Invited commentary

\title{
Making radiotherapy more efficient with FAIR data
}

\author{
Petros Kalendralis *, Matthijs Sloep, Johan van Soest, Andre Dekker, Rianne Fijten ${ }^{1}$ \\ Department of Radiation Oncology (Maastro), GROW School for Oncology, Maastricht University Medical Centre+, 6229 ET Maastricht, The Netherlands
}

\section{A R T I C L E I N F O}

\section{Keywords:}

Radiotherapy

FAIR data

Artificial intelligence

\begin{abstract}
A B S T R A C T
Given the rapid growth of artificial intelligence (AI) applications in radiotherapy and the related transformations toward the data-driven healthcare domain, this article summarizes the need and usage of the FAIR (Findable, Accessible, Interoperable, Reusable) data principles in radiotherapy. This work introduces the FAIR data concept, presents practical and relevant use cases and the future role of the different parties involved. The goal of this article is to provide guidance and potential applications of FAIR to various radiotherapy stakeholders, focusing on the central role of medical physicists.
\end{abstract}

Numerous data science advancements have been made in the radiotherapy domain, such as the extended incorporation of patients' imaging data for treatment purposes and the development of outcome prediction models. These were made possible by including high quality data consisting of information of patients, their treatment and follow-up.

With the rapid introduction of new technologies in radiotherapy, such as artificial intelligence (AI) and machine learning (ML), data driven approaches could influence the way patients are treated. Imagebased, biological, dosimetric and clinical variables can be combined with ML techniques to predict radiotherapy tumor outcomes [1-3] and toxicities [4]. However, radiotherapy data are highly complex and thus require clear definition-terminologies to ensure accessibility and interoperability (i.e. understandable by both machines and humans). Datasets without detailed, formal, standardized and applicable terminologies that enforce relationships within the data elements (i.e. ontologies) cannot be interpreted by others without expert knowledge of that specific dataset. As a result, many existing radiotherapy datasets are not reusable due to the absence of these ontological items.

This problem stems from barriers in the exchange of health data due to administrative, political, ethical and technical issues. For instance, inconsistencies in labeling and nomenclature of anatomical structure names in radiotherapy structures sets (RTSTRUCTs) are common. For example, when validating a published radiomics model, researchers discover a variety of label names for the Gross Tumor Volume (GTV), making the correct GTV selection problematic.

Aiming also to overcome problems like these, we need to implement the FAIR (Findable, Accessible, Interoperable, Reusable) data principles [5]. With this commentary article we would like to present our educational opinion based in our research findings and experience with the implementation of the FAIR data principles, rather than giving an exhaustive view of the FAIR principles. Furthermore, this manuscript is intended to provide an overview of the challenges and opportunities of implementing the FAIR principles in radiotherapy, highlighting the medical physicists' role. It ends with a suggested framework to develop responsible FAIR radiotherapy research.

FAIR stands for Findable, Accessible, Interoperable and Reusable (Fig. 1). Since its first publication [5] in 2016, the FAIR principles have been adopted by many institutes and research organizations worldwide $[6,7]$ and applied in a variety of disciplines [8,9].

Findability of data refers to a detailed description of metadata, indexed in a searchable source. Each dataset should be assigned a unique and persistent identifier for their unequivocal reference and citation. For instance, the publicly available NCSLC-Radiomics dataset [10] can be cited and referenced with its own unique identifier such as the Uniform Resource Identifier (URI).

Accessible data means that data are readable by both humans and computers with the appropriate authorization. Data should be stored in a trusted repository with an open protocol. Examples of trusted public repository for radiotherapy datasets case are the Cancer Imaging Archive (TCIA) [11], the Extensible Neuroimaging Archive Toolkit (XNAT) [12] hosted within the Dutch national research infrastructure [13], Dryad [14] and Zenodo [15]. It is important to mention that accessible data are not open data without constraint, it means that humans and machines may have access to them by respecting clear rules.

Interoperability refers to the use of formal, universal and broadly

\footnotetext{
* Corresponding author.

E-mail address: petros.kalendralis@maastro.nl (P. Kalendralis).

1 Senior author.
} 
applicable languages for knowledge sharing and representation, such as public ontologies. Ontologies are terminologies that give a meaning to the essential relationships between different data concepts. Typical examples of these languages are the Radiation Oncology Ontology (ROO) $[16,17]$, specifically designed for the radiotherapy domain, the Radiation Oncology Structures (ROS) [18] ontology and the Biomedical Imaging Methods (FBbi) [19] ontology. Similar interoperability-enhancing initiatives have been and continue to be undertaken by professional societies [20].

For data to be Reusable, researchers need to include detailed documentation and rich metadata. Publicly available reports about the acquisition, processing and origin of the data combined with a detailed description of technical details such as statistical analysis methods in a format of publicly available codes/algorithms, are highly recommended.

Despite its advantages, the FAIR approach has not yet been widely adopted in the radiotherapy domain due to various barriers. First, data preparation is costly, both monetary and labor intensive. There is an emerging need for novel IT solutions that bridge the gap between the need to "FAIRify" data for research purposes and every-day use of Electronic Health Record (EHR) systems, treatment planning systems and medical image storage archives. The attachment of ontologies for each data element recorded in the EHR systems to clearly establish their definition is an example.

Because each hospital uses various data sources at the same time, there is a vast amount of multi-source data that are mostly unstructured and not integrated. Its transformation into a valuable source of knowledge is therefore problematic due to the lack of interoperability between the different data sources. This, in combination with the unstructured nature of multiple sources, underlies the urgent need to introduce the FAIR data principles.
Moreover, as the radiotherapy data encrypt "sensitive" personal patients' information, there are ethical and security barriers regarding their use and property. There is an urgent need for a higher level of security that has to be taken into account as it is fundamental to ensure that patient data are protected. The different national regulations of each institute for data use decelerate the FAIR concept. Furthermore, the implementation of the FAIR data principles in compliance with the mandatory General Data Protection Regulation (GDPR) [21] is challenging. Specifically, the GDPR [21] includes the right of data transfer between social networks that prerequisite FAIR data. Contrarily, the GDPR does not allow data sharing between different institutions without a definitive purpose for research or other enterprise activities.

Additionally, the "FAIRification" process requires technical programming skills, which not all clinicians are familiar or proficient with. As a consequence, its adoption and implementation can be difficult as the short- and long-term return or reward are obscure to many.

Several other challenges and barriers for the implementation of the FAIR principles that are not mentioned in our study are described extensively by (inter)national guidelines such as the final report and action plan on FAIR data from the European Commission [22], academic publications such as the study of Jacobsen et al. [23] and non profit organizations initiatives like the Go FAIR foundation [24].

The FAIR principles have the potential to tackle the interoperability and reusability issues, using publicly available ontologies for radiation oncology and radiotherapy [25] such as the ROO [16,17] and other semantic technologies [26,27], such as the Resource Description Framework (RDF) [28]. Combining these two components results in a semantic data model. Semantic models are used to represent relationships between multiple concepts in the data. RDF represents the data in something called triples. Triples are composed of a subject ("patient"), a predicate ("has biological sex") and an object ("gender") that link the

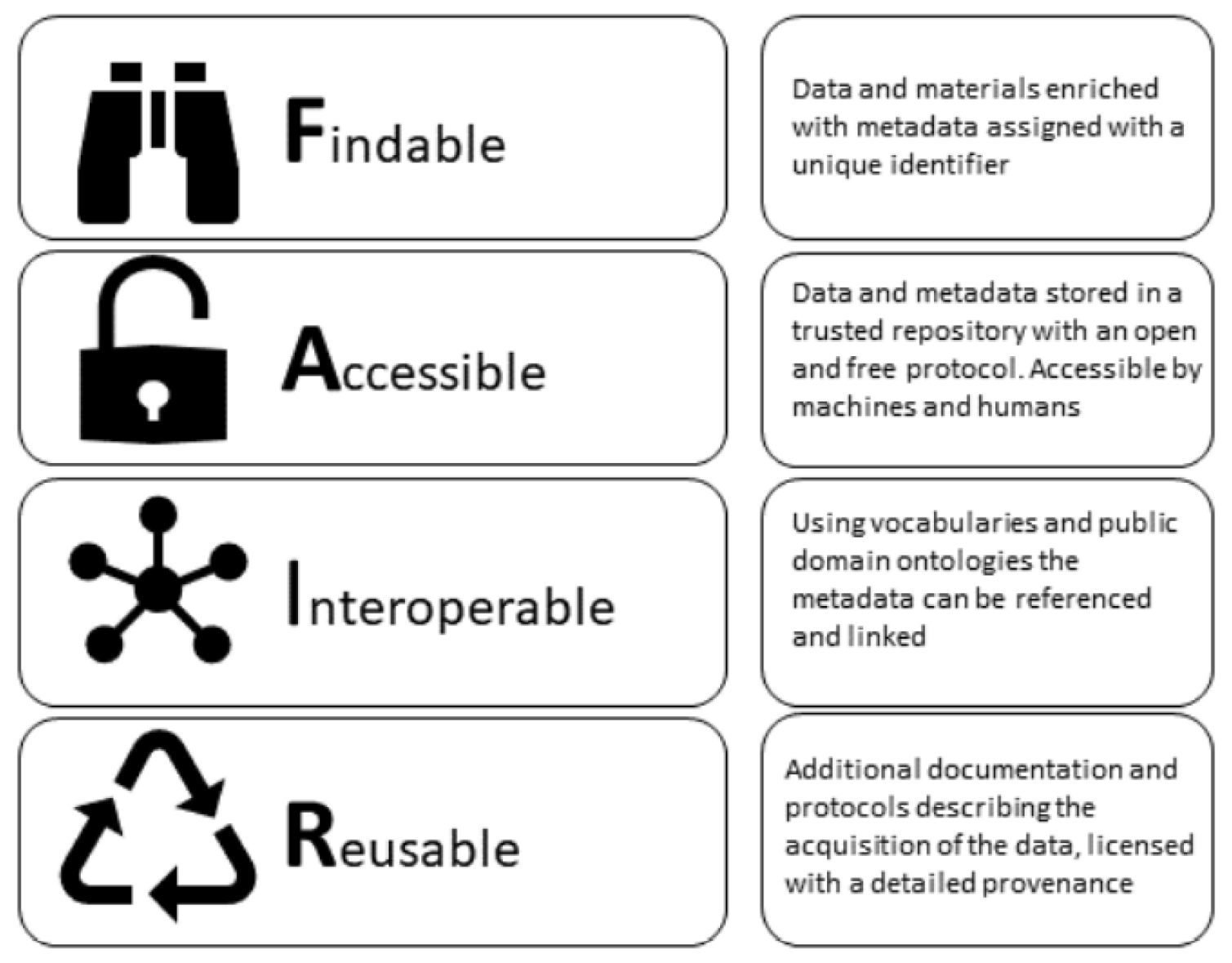

Fig. 1. Schematic representation and description of the FAIR data principles. 
relationships between the data items of a dataset. Each of these elements of a triple need to be defined by a publicly available ontological identifier. Fig. 2 displays the relationship between a patient and their gender in triple form.

By applying the FAIR principles to radiotherapy data, algorithms can assist the users in processing and manipulating data. As a consequence, $\mathrm{AI} / \mathrm{ML}$ implementation could be facilitated in numerous applications with large potentials in sparing resources in time-consuming repetitive procedures. A relevant example concerns the automatic delineation of the anatomical structures for treatment planning.

Furthermore, the implementation and adoption of the FAIR data principles ensure that research results and outputs can be exchanged and shared among different institutions. One of the potential applications that FAIR data enable in the radiotherapy domain, is the radiotherapy outcomes prediction modelling (exchange and validation) among different institutes exchanging model's parameters instead of patients' data [31].

FAIR compliant datasets have the potential to tackle the interoperability issues between the researchers. A representative example is the set of collections hosted by the above mentioned TCIA provided by Kalendralis et al. [32]. This study provided the clinical metadata, quantitative imaging features and DICOM metadata from four radiotherapy datasets mainly used for radiomics studies [33,34], in RDF [28] format using public ontologies $[16,17,29]$.

Besides the benefits of the establishment of collaborations between different institutes/clinics, one of the basic and valuable achievements of the FAIR principles for radiotherapy researchers and clinicians is the increase of the scientific outcome and of its impact on the community thanks to the largely improved generalizability and usability of the published results: and this is expected to increase the visibility of their work. Of note, publications associated with FAIR compliant data sharing are cited 69\% more frequently [35].

The incorrect implementation of the FAIR principles might have significant risks such as the "abuse" of data use by private interests. These risks can be prevented by the implementation of the FAIR principles as the data usage as the FAIR data users can make them accessible ensuring authentication and authorisation steps.

The generation of multisource data, with the goal to make advantage of it by reusing the data besides the immediate care of the patient, requires the inclusion of different stakeholders in the radiotherapy domain. It is important to identify and define the different stakeholders and their action items, establishing a shared responsibility relationship.

These stakeholders in the FAIR radiotherapy domain are radiation oncologists, radiologists (and other clinicians experts in imaging applications), medical physicists, researchers, radiotherapy enterprises (such as EHR or radiotherapy treatment planning systems companies), patients, IT personnel and managerial boards.

High-quality FAIR-compliant data will have a positive impact on the robustness of the clinical decisions for the patients with the prerequisite of a shared vision between the stakeholders. Our suggestion for their roles and responsibilities are shown in Table 1.

The definition of valuable clinical questions from the clinicians is crucial for the development and implementation of beneficial FAIR data
Table 1

Stakeholders of FAIR data in radiotherapy with the action items for them.

\begin{tabular}{ll}
\hline Stakeholders & Role definition \\
\hline Clinicians & - Influence the hospital boards to provide resources/ \\
& funding for the FAIR principles implementation with \\
& their clinical knowledge \\
Medical Physicists & - Data managers \\
& - Data collection and curation tasks \\
& - Commissioning of FAIR data tools \\
Researchers & - Clinical orientated FAIR research \\
& - Collaborate with doctors and physicists during data \\
Companies in & curing and conceptualizations of data usage \\
radiotherapy market & - FAIR-friendly tools \\
IT personnel & - Crofessionalize FAIR data infrastructure tools \\
& - the incorpo-ration of the FAIR principles into the daily \\
& - Adapting/tailoring infrastructures \\
Patients & - Understand the benefits of FAIR data \\
Hospital board members & - Data governance plan \\
& - Legal interoperability \\
& - Resources/funding
\end{tabular}

driven decisions into the clinic. Toxicity reduction to the organs at risk (OAR) during radiotherapy, improvement of the patients' cure rates, time and resources optimization are some of the interesting endpoints that can be evaluated prior to the development of a FAIR data tool in the clinic. Using their clinical knowledge, clinicians should take the role of ambassadors to influence the hospital board members to invest resources/funding to FAIR data driven clinical decisions for a personalized treatment approach of the patients.

Medical physicists can be considered as "data managers" as they usually have the database privileges and rights to acquire and extract the data in collaboration with the IT experts. Their role of facilitator is highly crucial and will be focused on later. Furthermore, they can commission new technologies for use in the clinic. As a result, they are typically assigned to the labor-intensive task of data curation and selection for patient care.

Researchers are assigned with the task of presenting the value of FAIR data by executing clinically oriented research. In most of the cases they may overlap and collaborate with the medical physicists and doctors involved in the process of data curation and conceptualizations regarding how to use these data for the development of new valuable knowledge. In the field of translational research, the involvement of both clinical and basic researchers will contribute a significant value in radiotherapy.

During the past years, various funding agencies and organizations globally encouraged researchers to submit proposals including the implementation of the FAIR data principles $[6,7,36]$.

A crucial bridge between the research and clinic are the radiotherapy market companies that can introduce products and applications related to FAIR data principles. The professionalization of FAIR data infrastructure tools should in future be included in the portfolios of the radiotherapy vendors. FAIR-inclusion criteria for patients cohorts

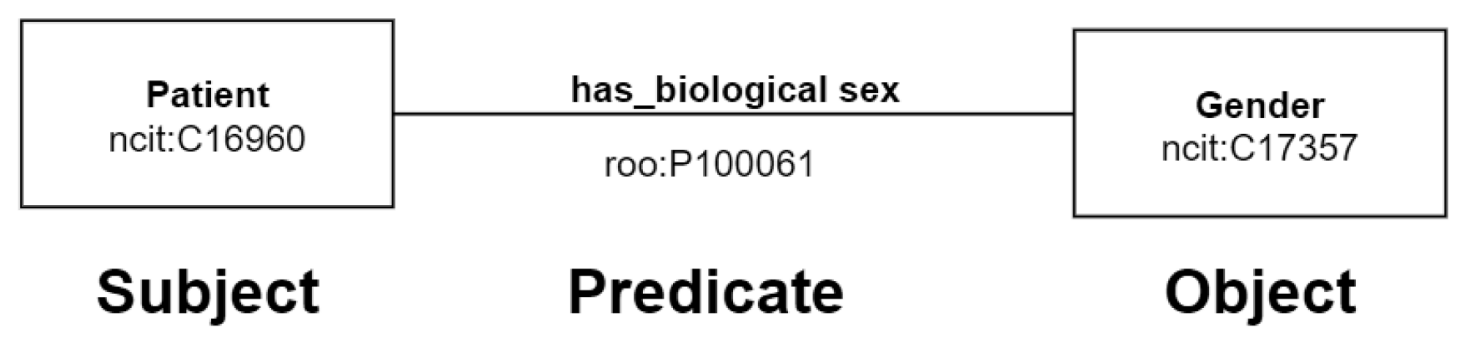

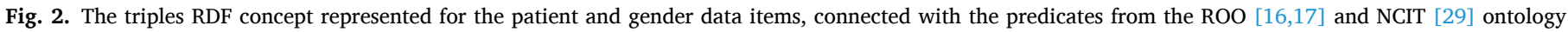
codes available in the Bioportal [30]. 
discovery, public repositories including radiotherapy outcomes prediction models, annotated applications with the visualization/registration of patients characteristics [37] and platforms that contain different imaging/treatment protocols from different centers are some of the examples of FAIR compliant applications that can be adopted by vendors in the radiotherapy market.

The need for a closer collaboration between the clinicians and IT professionals in the clinic is emerging for the introduction of the FAIR principles in daily practice. Important knowledge of data structures, data models and clinical databases schemas is held by IT personnel of the clinics and the implementation of a standardized data acquisition and usage plan should be established together with them. Moreover, IT professionals should be assigned the role of adapting and tailoring data infrastructures to fit the needs of a particular center.

Additionally, protocols regarding data reuse requests including approval from the institutional review board (IRB) of each institute should include clear steps describing the action points that should be taken from the different parties that would like to use patients' data and the further procedures that have to be followed for the acquisition of the data in the right format. This should include describing data access principles and protocols for outside parties to gain access to the data. Specifically, being informed about the benefits of FAIR data, the patients' role includes the task of giving their consent/approval to use their data.

The implementation of the FAIR principles should include leadership of each department and data governance policy should be established by such management/board members. Data lifecycle and stewardship plans that include the FAIR principles need to be as compulsory for research proposals as the data analysis protocol. Furthermore, assessment frameworks that include the certification of FAIR services provided should be implemented to the radiotherapy departments in combination with data science and data stewardship training curriculums/programs. Moreover, in the radiotherapy domain we deal with patients' sensitive information so there is a higher level of security that has to be taken into account as it is fundamental to ensure that patient data are protected.

As it is mentioned above, the medical physicists have the role of facilitator as they have the reputation of introducing and implementing novel technologies such as AI into the clinic. Although a leading and active role in the AI field is beneficial for the future career of medical physicists, they need to be included in a working group of multidiscipline experts. Closer collaboration with data scientists and computer scientists is more than necessary for the safe and sufficient development of AI technologies such as the FAIR concept, enabling access to high quality curated datasets needed for AI applications [38]. For their effective and constructive contribution of the medical physicists to this new emerging field in radiotherapy advanced data science skills (AI, data analysis, statistics) should be included in their educational curriculums and training schemes $[38,39]$.

In this manuscript, we made an introduction of the FAIR principles presenting the challenges and opportunities arising from their implementation to the radiotherapy data. Specific efforts and actions points are summarized and underlined from the stakeholders' perspective. In order to continue providing high quality personalized radiotherapy services, the stakeholders of the radiotherapy community should develop a close collaboration with each other trying to overcome and cover technological barriers. To further support that, it is desirable to include the FAIR data principles related topics in the educational schemes of the international medical physicists associations. Medical physics' new trend is correlated with the challenging AI field and the related professions should cope with this including establishing a clear data governance framework in radiotherapy departments.

\section{Declaration of Competing Interest}

The authors declare that they have no known competing financial interests or personal relationships that could have appeared to influence the work reported in this paper.

\section{References}

[1] Chao H-H, Valdes G, Luna JM, et al. Exploratory analysis using machine learning to predict for chest wall pain in patients with stage I non-small-cell lung cancer treated with stereotactic body radiation therapy. J Appl Clin Med Phys 2018;19(5): 539-46. https://doi.org/10.1002/acm2.12415.

[2] Valdes G, Solberg TD, Heskel M, Ungar L, Simone CB. Using machine learning to predict radiation pneumonitis in patients with stage I non-small cell lung cancer treated with stereotactic body radiation therapy. Phys Med Biol 2016;61(16): 6105-20. https://doi.org/10.1088/0031-9155/61/16/6105.

[3] Gennatas ED, Wu A, Braunstein SE, et al. Preoperative and postoperative prediction of long-term meningioma outcomes. PLoS One 2018;13(9):e0204161. https://doi. org/10.1371/journal.pone.0204161.

[4] Isaksson LJ, Pepa M, Zaffaroni M, et al. Machine learning-based models for prediction of toxicity outcomes in radiotherapy. Front Oncol 2020;10:790. https:// doi.org/10.3389/fonc.2020.00790.

[5] Wilkinson MD, Dumontier M, Aalbersberg IJ, et al. The FAIR guiding principles for scientific data management and stewardship. Sci. Data 2016;3. https://doi.org/ 10.1038/sdata.2016.18.

[6] European Commission. Horizon 2020, Work Programme 2018-2020, Health, demographic change and wellbeing, https://ec.europa.eu/research/participants/ data/ref/h2020/wp/2018-2020/main/h2020-wp1820-health_en.pdf [accessed 26 January 2021].

[7] Notice announcing funding opportunity issued for the NIH data commons pilot phase. Published June 23, 2017, https://grants.nih.gov/grants/guide/notice-files/ NOT-RM-17-031.html [accessed 26 January 2021].

[8] Lannom L, Koureas D, Hardisty AR. FAIR data and services in biodiversity science and geoscience. Data Intell 2020;2(1-2):122-30. https://doi.org/10.1162/dint_a 00034.

[9] Hiebel G, Goldenberg G, Grutsch C, Hanke K, Staudt M. FAIR data for prehistoric mining archaeology. Int J Digit Libr 2020. https://doi.org/10.1007/s00799-02000282-8 [Published online January 2].

[10] Aerts HJWL, Wee L, Rios Velazquez E, Leijenaar RTH, Parmar C, Grossmann P, et al. Data from NSCLC-radiomics. Cancer Imag Arch 2019. https://doi.org/ 10.7937/K9/TCIA.2015.PF0M9REI.

[11] Clark K, Vendt B, Smith K, et al. The cancer imaging archive (TCIA): Maintaining And Operating A Public Information Repository. J Digit Imaging 2013;26(6): 1045-57. https://doi.org/10.1007/s10278-013-9622-7.

[12] Marcus DS, Olsen TR, Ramaratnam M, et al. The extensible neuroimaging archive toolkit. Neuroinformatics 2007;5:11-33. https://doi.org/10.1385/NI:5:1:11.

[13] Dutch national research infrastructure TraIT, www.ctmm-trait.nl [accessed 26 January 2021].

[14] Dryad repository, https://datadryad.org/stash [accessed 26 January 2021].

[15] Zenodo repository, https://zenodo.org/ [accessed 26 January 2021].

[16] Traverso A, van Soest J, Wee L, Dekker A. The radiation oncology ontology (ROO): Publishing linked data in radiation oncology using semantic web and ontology techniques. Med Phys 2018;45(10):e854-62. https://doi.org/10.1002/mp.12879.

[17] Radiation Oncology Ontology (ROO), https://bioportal.bioontology.org/ontol ogies/ROO [accessed 26 January 2021].

[18] Radiation Oncology Structures (ROS) Ontology, https://bioportal.bioontology org/ontologies/ROS [accessed 26 January 2021].

[19] Biomedical Imaging Methods (FBbi) Ontology, https://bioportal.bioontology.org/o ntologies/FBbi [accessed 26 January 2021]

[20] Mayo CS, Moran JM, Bosch W, et al. American Association of Physicists in Medicine Task Group 263: standardizing nomenclatures in radiation oncology. Int J Radiat Oncol Biol Phys 2018;100(4):1057-66. https://doi.org/10.1016/j. ijrobp.2017.12.013.

[21] General Data Protection Regulation (GDPR), https://gdpr-info.eu/ [accessed 26 January 2021].

[22] Final Report And Action Plan From the European Commission Expert Group on FAIR Data, "Turning FAIR into reality." https://ec.europa.eu/info/sites/info/files /turning_fair_into_reality_0.pdf [accessed 26 January 2021].

[23] Jacobsen A, de Miranda Azevedo R, Juty N, et al. FAIR principles: interpretations and implementation considerations. Data Intell 2020;2(1-2):10-29. https://doi. org/10.1162/dint_r_00024.

[24] Go FAIR non profit organisation, https://www.go-fair.org/ [accessed 26 January 2021].

[25] Min H, Manion FJ, Goralczyk E, Wong Y-N, Ross E, Beck JR. Integration of prostate cancer clinical data using an ontology. J Biomed Inform 2009;42(6):1035-45. https://doi.org/10.1016/j.jbi.2009.05.007.

[26] Van Soest J, Lustberg T, Grittner D, et al. Towards a semantic PACS: using semantic web technology to represent imaging data. Stud Health Technol Inform 2014;205: 166-70.

[27] Alonso-Calvo R, Perez-Rey D, Paraiso-Medina S, Claerhout B, Hennebert P, Bucur A. Enabling semantic interoperability in multi-centric clinical trials on breast cancer. Comput Methods Programs Biomed 2015;118(3):322-9. https://doi.org/ 10.1016/j.cmpb.2015.01.003.

[28] Resource Description Framework (RDF), https://www.w3.org/RDF/ [accessed 26 January 2021].

[29] National Cancer Institute Thesaurus Ontology, https://bioportal.bioontology.org/o ntologies/NCIT [accessed 26 January 2021].

[30] Bioportal website, https://bioportal.bioontology.org/ [accessed 26 January 2021]. 
[31] Deist TM, Dankers FJWM, Ojha P, et al. Distributed learning on 20,000+ lung cancer patients - the personal health train. Radiother Oncol 2020;144:189-200. https://doi.org/10.1016/j.radonc.2019.11.019.

[32] Kalendralis P, Shi Z, Traverso A, et al. FAIR-compliant clinical, radiomics and DICOM metadata of RIDER, interobserver, Lung1 and head-Neck1 TCIA collections. Med Phys 2020. https://doi.org/10.1002/mp.14322. mp.14322.

[33] Aerts HJWL, Velazquez ER, Leijenaar RTH, et al. Decoding tumour phenotype by noninvasive imaging using a quantitative radiomics approach. Nat Commun 2014; 5(1):4006. https://doi.org/10.1038/ncomms5006.

[34] Lambin P, Leijenaar RTH, Deist TM, et al. Radiomics: the bridge between medical imaging and personalized medicine. Nat Rev Clin Oncol 2017;14(12):749-62. https://doi.org/10.1038/nrclinonc.2017.141.
[35] Piwowar HA, Day RS, Fridsma DB. Sharing detailed research data is associated with increased citation rate. Ioannidis J, ed. PLoS One 2007;2(3):e308. https://doi. org/10.1371/journal.pone.0000308.

[36] 2016 National Research Infrastructure, https://www.dese.gov.au/resource s/2016-national-research-infrastructure-roadmap [accessed 26 January 2021].

[37] Gopinath D, Agrawal M, Murray L, Horng S, Karger D, Sontag D. Fast, structured clinical documentation via contextual autocomplete. arXiv e-prints, arXiv-2007; 2020.

[38] Fiorino C, Jeraj R, Clark CH, et al. Grand challenges for medical physics in radiation oncology. Radiother Oncol 2020;153:7-14. https://doi.org/10.1016/j. radonc.2020.10.001.

[39] Thompson RF, Valdes G, Fuller CD, et al. Artificial intelligence in radiation oncology: a specialty-wide disruptive transformation? Radiother Oncol 2018;129 (3):421-6. https://doi.org/10.1016/j.radonc.2018.05.030. 